\title{
Quantifying the Impact of Comorbidities on Outcomes Following Surgery for Osteoporotic Vertebral Compression Fractures
}

\author{
Anmol Gupta ${ }^{1}$ Thomas Cha ${ }^{1}$ Joseph Schwab ${ }^{1}$ Harold Fogel $^{1}$ Daniel Tobert ${ }^{1}$ Afshin E. Razi \\ Carl Paulino $^{3}$ Christopher M. Bono ${ }^{1} \quad$ Stuart Hershman ${ }^{1}$
}

${ }^{1}$ Department of Orthopaedics, Icahn School of Medicine at Mount Sinai, The Mount Sinai Hospital, Massachusetts General Hospital, Boston, Massachusetts, United States

2Department of Orthopaedics, Maimonides Bone and Joint Center, Maimonides Medical Center, Brooklyn, New York, United States

${ }^{3}$ Department of Orthopaedic Surgery, SUNY Downstate Health Sciences University, NYP Brooklyn Methodist Hospital, Brooklyn, New York, United States

J Clin Interv Radiol ISVIR 2021;6:3-9.

\begin{abstract}
Address for correspondence Stuart Hershman, MD, Department of Orthopaedics, Massachusetts General Hospital, 55 Fruit Street, Boston, MA 02114, United States

(e-mail: shhershman@mgh.harvard.edu).
\end{abstract}

\section{Abstract}

Keywords

- mortality

- vertebral compression fracture

- osteoporosis

- cement augmentation
Introduction Studies have shown that osteoporotic patients are more likely to have medical or surgical complications postoperatively. In this study, we determine the predictive value of various comorbidities on the likelihood of postoperative complications, mortality, and 30-day readmission following cement augmentation for osteoporotic vertebral compression fractures (OVCFs).

Materials and Methods A retrospective analysis of the American College of Surgeons National Surgery Quality Improvement Project (ACS-NSQIP) database from 2007 to 2014 identified 1979 patients who met inclusion criteria. A multivariate logistic regression analysis was utilized to determine the relationship between various comorbidities and perioperative mortality, postoperative complications, and 30-day readmission rates. Results A history of cerebrovascular accident (CVA), coagulopathy, diminished preoperative functional status, and/or an American Society of Anesthesiologists (ASA) class $>2$ were statistical predictors of postoperative complications. CVA generated the highest odds ratio among these comorbidities ( $O R=5.36, p=0.02$ for minor complications; $\mathrm{OR}=4.60 p=0.05$ for major complications). Among the 15 comorbidities considered, steroid use $(\mathrm{OR}=1.81 ; p=0.03)$ and an ASA class $>2(\mathrm{OR}=14.65 ; p=0.01)$ were the only ones that were correlated with mortality; an ASA class $>2$ had a particularly strong effect on the likelihood of mortality $(O R=14.65)$. Chronic obstructive pulmonary disorder (COPD), obesity, significant weight loss, and an ASA class $>2$ were correlated with 30-day readmissions. Congestive heart failure (CHF), diabetes, dialysis, hypertension, or smoking was not correlated with adverse postoperative outcomes.

Conclusion of the 15 comorbidities considered in this study, four were statistically associated with increased rates of postoperative complications, two were associated with increased mortality, and four were associated with increased rates of readmission at 30 days. The presence of CHF, diabetes mellitus (DM), hypertension, ascites, renal failure, or smoking were not associated with the adverse outcomes studied.

Level of Evidence III. published online May 18, 2021
DOI https://doi.org/ $10.1055 / \mathrm{s}-0041-1729466$ ISSN 2457-0214
(C) 2021. Indian Society of Vascular and Interventional Radiology. This is an open access article published by Thieme under the terms of the Creative Commons Attribution-NonDerivative-NonCommercial-License, permitting copying and reproduction so long as the original work is given appropriate credit. Contents may not be used for commercial purposes, or adapted, remixed, transformed or built upon. (https://creativecommons.org/licenses/by-nc-nd/4.0/). Thieme Medical and Scientific Publishers Pvt. Ltd. A-12, 2nd Floor, Sector 2, Noida-201301 UP, India 


\section{Introduction}

Each year, over two million fragility fractures occur in the United States, hospitalizing over 500,000 elderly patients and costing the health care system over $\$ 5.1$ billion annually. ${ }^{1,2}$ Among these fragility fractures, roughly one third are osteoporotic vertebral compression fractures (OVCFs). ${ }^{3}$ OVCFs commonly cause dysfunction, severe pain, and postural changes that may be treated via cement augmentation procedures. ${ }^{4,5}$ Considering the rising incidence of osteoporotic fractures in the population ${ }^{6}$ and the frailty commonly associated with OVCF patients, ${ }^{7}$ literature regarding the clinical management of this condition is becoming progressively more relevant. Treatment options for OVCF include benign neglect, bracing, medication, and procedures such as cement augmentation. While the treatment plan for a given patient is based on various clinical factors, several studies have shown that vertebral cement augmentation may offer better long-term pain relief than medical management alone for a subset of patients. ${ }^{8-11}$ However, prior to recommending an invasive procedure, clinicians must consider the risk-profile of each patient. In this study, we attempt to determine the relationship between 15 comorbidities and the likelihood of developing complications, mortality, or 30-day readmission following an invasive procedure for an OVCF. In doing so, we hope to help guide clinical decision-making regarding treatment plans and help clinicians manage patients' expectations following vertebral cement augmentation.

\section{Materials and Methods}

In this retrospective study, data from the American College of Surgeons National Surgery Quality Improvement Project (ACS-NSQIP) from 2007 through 2014 was reviewed. Because information within this database is deidentified, this study was exempt from institutional review board (IRB) approval. Following a methodology utilized by others, ${ }^{12-14}$ patients who sustained an OVCF in the lumbar or thoracic region of the spine were identified using current procedural terminology (CPT) codes and International Classification of Disease (ICD-9 or ICD-10) codes. ICD-9 codes 733.13, 805.2, and 805.4 (utilized for the years 2007-2013) and ICD-10 codes M48.56XA, S22.009A, S22.068A, and S22.089A (used for 2014) were also included in this study. To ensure all target patients in the ACS-NSQIP database were captured, patients assigned any of the following CPT codes were also included: 22510, 22511, $22512,22513,22514$, or 22515; these CPT codes refer to vertebral cement augmentation procedures.

Patients with an OVCF in the cervical, sacral, or unclassified region of the spine, and/or those with confirmed spinal or central nervous system (CNS) tumors were excluded. For purposes of conducting a logistic regression analysis, the following patient characteristics were considered: gender, body mass index (BMI), functional status prior to procedure, preoperative comorbidities and preoperative serum albumin level, American Society of Anesthesiologists (ASA) status, postoperative complications, mortality, reoperations, and 30-day readmissions. If greater than 10 percent of patients were missing data for a given variable, that variable was excluded from the study.

Outcome measures for this study included minor postoperative complications, major postoperative complications, patient mortality, 30-day readmission due to any cause, and 30-day readmission related to OVCF. As in a previous study by Chung et al, ${ }^{14}$ the following issues were considered minor postoperative complications: pneumonia, urinary tract infection (UTI), deep vein thrombosis (DVT), or incision site complications. Major postoperative complications included cardiac arrest, acute myocardial infarction (MI), sepsis, septic shock, stroke, pulmonary embolism (PE), acute renal failure, a coma lasting more than 24 hours, and reintubation.

\section{Statistical Analysis}

Using multivariate logistic regression, odds ratios (OR) with corresponding $p$ values and 95\% confidence intervals (CI) were calculated. This statistical tool was used to determine the direct correlation between 15 comorbidities and primary outcome measures, while also accounting for possible confounders such as age, gender, or preoperative serum albumin levels. While most comorbidities were considered dichotomous, preoperative functional status was treated as a categorical variable. Patients exhibiting an inability to perform at least one activity of daily living (ADL) were considered partially dependent; if a patient was unable to perform any ADLs, he or she was classified as completely dependent. Preoperative serum albumin level was treated as a continuous variable. To determine which variables should be utilized in the logistic regression, a series of bivariate analyses between age and the variable in question were conducted; those that generated $p$ values less than 0.05 were included in the multivariate analysis. Any variable for which fewer than five incidences occurred within the patient dataset was excluded. For statistical purposes, a patient listed as greater than 90 years of age in the ACS-NSQIP database was treated as a 90 -year old.

\section{Results}

Upon application of inclusion and exclusion criteria, 1979 patients were found in the ACS-NSQIP database. - Table 1 provides a demographic overview of our study population. Patients were on average approximately 74 years of age at the time of vertebral cement augmentation. More than two-thirds were female and the average BMI was slightly under 27. Approximately one out of seven patients had a smoking history within 1 year of the index procedure; the same was true regarding chronic steroid use and diminished functional status prior to intervention. On average, patients had a preoperative albumin in the healthy range $(>3.5){ }^{7}$

- Table 2 summarizes the prevalence of various comorbidities as well as the distribution of ASA status in the study population. Nearly two-thirds of patients had a history of hypertension and more than one-quarter were obese; almost $80 \%$ of patients had an ASA class of III or higher, highlighting the frailty of OVCF patients. - Table 3 summarizes postoperative outcomes in our study population. Mortality 
Table 1 Patient characteristics

\begin{tabular}{|l|l|l|}
\hline \multicolumn{2}{|l|}{ Total $(n=1979)$} & SD or \% \\
\hline Parameter & 73.92 & 10.82 \\
\hline Age (years) ${ }^{\text {a }}$ & 1328 & $67.1 \%$ \\
\hline Sex (female) & 26.74 & 6.45 \\
\hline BMI & 3.58 & 0.65 \\
\hline Mean preoperative albumin & 293 & $14.8 \%$ \\
\hline $\begin{array}{l}\text { Steroid use for chronic } \\
\text { condition }\end{array}$ & 270 & $13.6 \%$ \\
\hline Smoking history & 26 & $1.3 \%$ \\
\hline On dialysis & 1661 & $85.3 \%$ \\
\hline Functional status prior to surgery & 265 & $13.6 \%$ \\
\hline Independent & 22 & $1.1 \%$ \\
\hline Partially dependent & 31 & - \\
\hline Totally dependent & \multicolumn{2}{|l}{} \\
\hline Unknown &
\end{tabular}

Abbreviations: BMI, body mass index; SD, standard deviation.

${ }^{a}$ For statistical purposes, patients age $90+$ are assumed to be 90 years of age.

Table 2 Comorbidities and distribution of ASA status across 1979 patients

\begin{tabular}{|c|c|c|}
\hline Diagnosis & \# Patients & $\%$ \\
\hline \multicolumn{3}{|l|}{ Comorbidity } \\
\hline $\mathrm{CHF}$ & 56 & $2.8 \%$ \\
\hline COPD & 307 & $15.5 \%$ \\
\hline Coagulopathy & 173 & $8.7 \%$ \\
\hline CVA & 16 & $0.8 \%$ \\
\hline Diabetes & 385 & $19.5 \%$ \\
\hline Dialysis & 26 & $1.3 \%$ \\
\hline Hypertension & 1317 & $66.5 \%$ \\
\hline Ascites & 3 & $0.2 \%$ \\
\hline $\mathrm{Ml}<6$ months & 0 & $0.0 \%$ \\
\hline Obesity (BMI $\geq 30$ ) & 497 & $25.1 \%$ \\
\hline Renal Failure & 4 & $0.2 \%$ \\
\hline Smoker (w/in last year) & 270 & $13.6 \%$ \\
\hline Steroid use for chronic condition & 293 & $14.8 \%$ \\
\hline Weight loss & 66 & $3.3 \%$ \\
\hline \multicolumn{3}{|l|}{ ASA Status } \\
\hline ASA Class I & 11 & $0.6 \%$ \\
\hline ASA Class II & 390 & $19.7 \%$ \\
\hline ASA Class III & 1297 & $65.6 \%$ \\
\hline ASA Class IV & 274 & $13.9 \%$ \\
\hline ASA Class V & 4 & $0.2 \%$ \\
\hline None listed & 3 & - \\
\hline
\end{tabular}

Abbreviations: ASA, American Society of Anesthesiologists; BMI, body mass index; CHF, congestive heart failure; COPD, chronic obstructive pulmonary disorder; CVA, cerebrovascular accident; MI, myocardial infarction.

was seen in $4.6 \%$ of patients within 30 days of the index procedure, and $13.1 \%$ of patients were readmitted. Overall, $5.1 \%$ of patients experienced a major complication, most commonly sepsis and reintubation, and $7.2 \%$ of patients suffered minor complications.
Table 3 Postoperative outcomes among 1979 patients

\begin{tabular}{|c|c|c|}
\hline Diagnosis & \# Patients & $\%$ \\
\hline Mortality & 91 & $4.6 \%$ \\
\hline Reoperations & 83 & $4.2 \%$ \\
\hline Readmissions & 259 & $13.1 \%$ \\
\hline Readmission related to VCF & 169 & $8.5 \%$ \\
\hline Mean length of Hospital Admission & 5.93 & 13.89 \\
\hline \multicolumn{3}{|l|}{ Major complications } \\
\hline Acute MI & 7 & $0.4 \%$ \\
\hline Cardiac arrest & 10 & $0.5 \%$ \\
\hline Sepsis & 38 & $1.9 \%$ \\
\hline Septic shock & 18 & $0.9 \%$ \\
\hline Stroke & 10 & $0.5 \%$ \\
\hline PE & 16 & $0.8 \%$ \\
\hline Acute renal failure & 5 & $0.3 \%$ \\
\hline Coma $>24$ hours & 1 & $0.1 \%$ \\
\hline Reintubation & 31 & $1.6 \%$ \\
\hline Prolonged intubation (> 7 days) & 17 & $0.9 \%$ \\
\hline Overall & 101 & $5.1 \%$ \\
\hline \multicolumn{3}{|l|}{ Minor Complications } \\
\hline DVT & 31 & $1.6 \%$ \\
\hline Pneumonia & 64 & $3.2 \%$ \\
\hline UTI & 63 & $3.2 \%$ \\
\hline Surgical site complication & 39 & $2.0 \%$ \\
\hline Overall & 143 & $7.2 \%$ \\
\hline
\end{tabular}

Abbreviations: DVT, deep vein thrombosis; MI, myocardial infarction; PE, pulmonary embolism; UTI, urinary tract infection; VCF, vertebral compression fracture.

-Fig. 1 summarizes the results of our multivariate logistic regression. To determine which factors to integrate into the analysis, a series of bivariate tests were conducted. Of the 15 comorbidities recorded in our database, 11 bore a statistical impact ( $p$ value $<0.05$ ) on at least one of the primary outcome measures. These included a history of congestive heart failure (CHF), chronic obstructive pulmonary disease (COPD), coagulopathy, cerebrovascular accident (CVA), diabetes mellitus (DM), dialysis, obesity, long-term steroid use, weight loss exceeding $10 \%$ of body weight over the past 6 months, ASA class $>2$, and preoperative functional status. Ascites and renal failure had fewer than five incidences and therefore were excluded from analysis. In addition, hypertension and smoking were also excluded from the analysis because the bivariate analyses for these variables failed to demonstrate statistical significance. To further validate our results, age, gender, and preoperative serum albumin levels were also integrated in our analysis.

Results from - Fig. 1 show that preoperative functional status $(p=0.03$ and 0.05$)$, ASA class $>2(p=0.01)$, and/or a history of CVA $(p=0.02)$ were statistically correlated with minor complications. Preoperative functional status $(p<.01)$, 


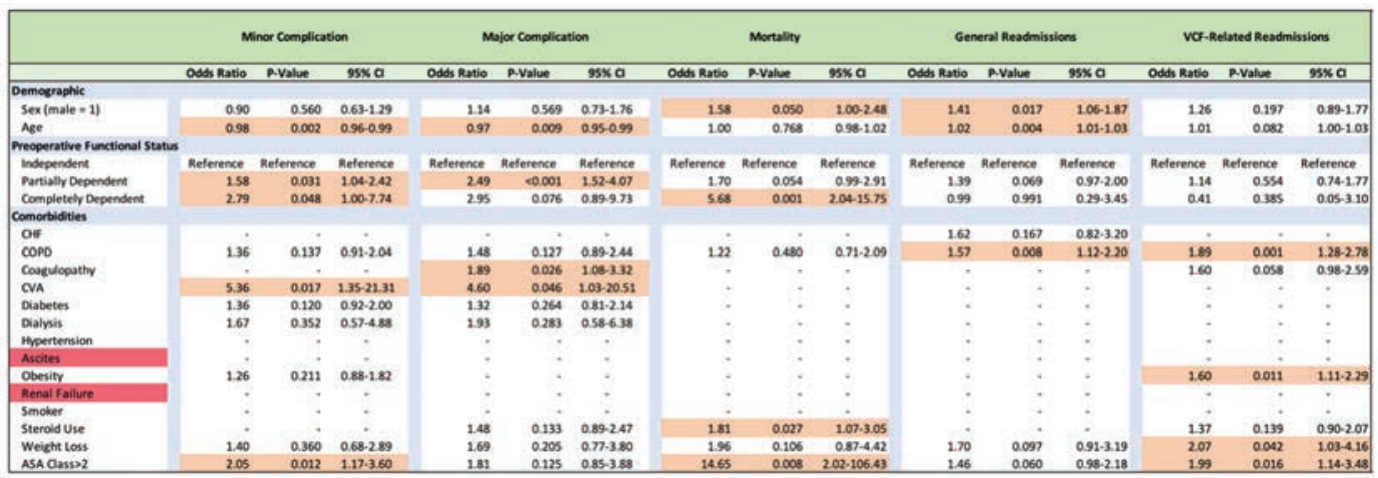

Fig. 1 Multivariate logistic regression analysis for minor complications, major complications, mortality, and 30-day readmissions. Variables with statistically significant p-values are highlighted in orange. Ascites and renal failure (highlighted in red) were excluded from logistic regression because of small sample size for these populations.

a history of CVA $(p=0.05)$, and a history of coagulopathy $(p=0.03)$ were associated with major complications. Notably, a history of CVA also exhibited the highest OR (5.36 and 4.60 , respectively) among any comorbidity considered in this study. Our data also show that preoperative functional status $(p<.01)$, chronic steroid use $(p=0.03)$, and an ASA class $>2(p<0.01)$ were statistically correlated with mortality; the latter exhibited the highest impact on mortality $(\mathrm{OR}=14.65)$. Readmission within 30 days of the index procedure was associated with a history of $\operatorname{COPD}(p<0.01)$, obesity $(p=0.01)$, significant weight loss $(p=0.04)$, and an ASA class $>2(p=0.02)$.

\section{Discussion}

As the most common type of fragility fracture, OVCFs occur every 22 seconds on average around the globe. ${ }^{15}$ The prevalence of OVCF is estimated to be $25 \%$ in postmenopausal women over age 50 , and $40 \%$ of women over age 80 , thus making the management of OVCF highly relevant to patient care and health care costs..$^{16-18}$ As a result of the high prevalence, demographics, interventions, and patient outcomes following procedures for OVCF have been well-studied. ${ }^{19-21}$ Toy et al found an association between postoperative complications and an unfavorable ASA class, in addition to inpatient status prior to procedure. ${ }^{22}$ Similarly, we found that patient classification by ASA status is in some regards a "catch-all" for the general health of a patient, ${ }^{23}$ helping clinicians identify whether a patient is healthy, has mild systemic disease, severe systemic disease, or life-threatening disease. ${ }^{24}$ To assign an ASA class to a patient, many of the comorbidities from this study are considered..$^{24,25}$ Categorizing a patient as "mildly sick" or "moderately sick," while helpful, may not give the specific insight that our data provides. The same can be said of preoperative functional status, which was included in our analysis to be as comprehensive as possible; however, like ASA status, it may not provide the specific insight that formally diagnosed medical conditions provide. Thus, the motivation for our logistic regression analysis was to see which disease processes were specifically impactful in the treatment of OVCF.
While we found that patient ASA class was associated with complications, we also identified correlations with a history of CVA and coagulopathy. These comorbidities are, not surprisingly, among those factors that would lead to an ASA classification greater than 2 . At the same time, other comorbidities that may also place patients in the same category, such as CHF or hypertension, were not found to be associated with any adverse outcomes following cement augmentation for OVCF. By providing this specific quantitative data from a large sample size, we hope to identify which comorbidities predict complications, mortality, or readmission, potentially enabling physicians to form a better treatment plan.

Our data shows that the most significant predictor of complications, both in terms of statistical significance and $\mathrm{OR}$, is a history of CVA ( $p=0.02$ and $\mathrm{OR}=5.36$ for minor complications; $p=0.05$ and $\mathrm{OR}=4.60$ for major complications). Coagulopathy was also associated with major postoperative complications ( $p=0.03$ and OR $=1.89$ ), however neither coagulopathy nor a history of CVA was found to be statistically correlated with mortality or readmission. To explain this, we considered the long-term treatment of CVA, which commonly involves antiplatelet or anticoagulant therapy to prevent reoccurrence. ${ }^{26,27}$ Prior to intervention, CVA patients often discontinue their anticoagulation medication. ${ }^{28}$ In an elderly and frail OVCF patient population, the lack of anticoagulation may disproportionately increase the risk of DVT postoperatively, which was considered a minor complication in our study. Additionally, a prior CVA may increase the likelihood of a second ischemic event following the procedure-this was considered a major complication. ${ }^{29,30}$ Our theory is supported by the literature, which suggests that $25 \%$ of CVA are recurrent, thereby increasing the likelihood of such an event in patients with a history of CVA. ${ }^{31}$

In terms of mortality, we found a statistical association in patients using steroids and those with ASA status $>2$. This correlation may be due to the association of steroids with sepsis and septic shock. While these complications are somewhat rare (1.9\% experienced sepsis and $0.9 \%$ endured septic shock), they are often life-threatening in the frail and elderly OVCF population. ${ }^{32,33}$ In one study relating to colorectal surgery, patients using steroids chronically were shown 
to have a higher risk of malnutrition, diabetes, bleeding disorders, and shock ${ }^{34}$; postoperatively, they exhibited higher rates of mortality and morbidity. ${ }^{34}$ Singla et al showed that following lumbar spine fusion, chronic steroid use was associated with incisional site infections in patients over the age of 65 as well as increased mortality rates. ${ }^{35}$ Our study corroborates this finding and highlights the potential perils of chronic steroid use in patients undergoing vertebral cement augmentation for OVCF.

A statistical correlation was seen in readmission rates in patients with obesity, COPD, and recent weight loss exceeding $10 \%$ of body weight. Ilyas et al also showed that obesity was associated with an increased rate of readmission following lumbar spine surgery, ${ }^{36}$ while Elsamadicy found a correlation between BMI and elective spine procedures in general. ${ }^{37}$ For this reason, it may be prudent for physicians to inform obese patients and those with COPD of the increased likelihood for readmission. Recent weight loss exceeding $10 \%$ of total body weight over 6 months is suggestive of a malignancy or significant infection-these patients may need to be examined further for the possibility of other pathology associated with these conditions, which could explain the higher rate of readmission. ${ }^{38}$

Gupta et $\mathrm{al}^{7}$ showed that preoperative albumin levels predict the incidence of postoperative complications following cement augmentation for OVCF and that the likelihood of an adverse outcome is proportional to the degree of hypoalbuminemia. To ensure the accuracy of results in this study, serum albumin level was treated as a continuous variable instead of a dichotomous variable. Similarly, our multivariate analysis included many of the various elements that comprise the Charlson comorbidity index (CCI). This included factors such as age, history of CHF, CVA, COPD, diabetes mellitus, and other comorbidities for which information was provided within the ACS-NSQIP database. The CCI is used to predict the risk of death within 1 year of hospitalization, based on comorbid conditions. The literature suggests that ASA status and CCI are related and often in close agreement, hence its relevance to this study. ${ }^{21,39,40}$ While this study does not control for all possible confounders, we felt that both CCI and preoperative serum albumin levels were important to incorporate in our analysis.

The ACS-NSIP database utilized in this study enabled us to analyze postprocedural outcomes following cement augmentation for the treatment of OVCF. However, we are unable to comment on how patients who are treated nonsurgically do in comparison to those who undergo cement augmentation based on our data. To address this alternative approach, we consider recent literature. Ong et al, in a 5-year study, showed that both balloon kyphoplasty and vertebroplasty were associated with statistically lower mortality rates in comparison to nonsurgical management of vertebral compression fracutres. ${ }^{41}$ Similarly, Hirsch et al utilized a 10 -year sample of Medicare data to conduct a number needed to treat analysis. This study showed that at both 1-year and 5-year follow-ups kyphoplasty patients did better. The adjusted number needed to treat to save 1 life from nonsurgical management versus kyphoplasty was 14.8 at year 1 and
11.9 at year $5 .^{42}$ Finally, Hinde et al, in a 2020 systematic review involving over two million patients, showed that patients who underwent vertebral augmentation procedures for treatment of OVCF were $22 \%$ less likely to expire 10 years postintervention. ${ }^{43}$ Thus, while some patients undergo complications from vertebral augmentation, for most people suffering from OVCF, it remains a stronger alternative to nonoperative management.

This study has multiple limitations. Our findings depend on the accuracy of deidentified spreadsheets from the ACS-NSQIP database without the possibility of verification through viewing original patient charts. Therefore, it is possible that patients may have been improperly included or excluded due to potential errors within the ACS-NSQIP database. While the NSQIP database is frequently utilized, ${ }^{44,45}$ there was no obvious way to correct for this. Furthermore, the NSQIP database does not provide information with regard to the specialty of the treating physician; it is possible that the outcomes of augmentation procedures conducted by interventional radiologists differ from those performed by surgeons or other subspecialists. It also is unable to provide information regarding adherence (or lack thereof) by clinicians to standardized care pathways such as SIR, SNIS, or the UCLA/Rand appropriateness method. These guidelines help ensure the standard of care is followed when treating patients.

From a more technical perspective, another challenge with the NSQIP database is its use of the term "null" instead of "no" when identifying patients with CNS tumors. While both terms are used throughout the database, the former term, in comparison to the latter, does not provide the same degree of certainty that a patient does not have a CNS tumor. In this study, both terms were considered acceptable for purposes of meeting inclusion criteria. Finally, variables recorded in ACS-NSQIP changed from year to year; periodically, some were added while others were removed. Although this constraint was carefully tracked and accounted for, it necessitated the exclusion of some variables from our regression analysis. Our analysis of 30-day readmission rates is also limited by the database. ACS-NSQIP defines a 30-day readmission from the date of the procedure and not the date of discharge ${ }^{46}$ Therefore, a sick patient discharged 3 weeks after the index procedure has only 9 days postdischarge to qualify for a 30-day readmission using this methodology. On the contrary, a patient discharged 2 days following an uncomplicated procedure has 28 days to be readmitted to be considered a 30-day readmission. This record-keeping limitation may have impacted our ability to fully assess the relationship between 30-day readmission and the comorbidities considered. Moreover, ACS-NSQIP does not track readmissions that occur after the initial 30-day period following a procedure.

Other limitations include the decision to avoid distinguishing between different procedural modalities. This study did not control for whether a patient underwent a multilevel or a single-level procedure in treating an OVCF. It also did not distinguish between cement augmentation techniques. In this database study, we relied on the clinical judgment of the clinician to identify which procedure would be best for a 
given patient, without controlling for the modality utilized. Future studies could compare other factors that may be statistically associated with adverse outcomes in the setting of OVCF, such as inpatient status prior to procedure or postoperative disposition. It may also be valuable to investigate the correlation between the factors considered in this study and other spine pathologies.

\section{Conclusion}

Data from this study may help clinicians identify comorbidities that can significantly affect outcomes. By quantifying the risk associated with these comorbidities, clinicians may be better equipped to guide the expectations of their patients and form treatment plans. Of the 15 comorbidities considered in this study, four were statistically associated with complications (preoperative functional status; ASA class > 2; history of coagulopathy; history of CVA), two were associated with increased rates of mortality (chronic steroid use; ASA class $>2$ ), and four were associated with increased rates of readmission (COPD; obesity; weight loss; ASA class >2). A history of CHF, DM, hypertension, ascites, renal failure, and smoking were not found to be associated with these adverse outcomes.

\section{Note}

The manuscript submitted does not contain information about medical device (s)/drug (s). No funds were received in support of this work. No benefits in any form have been or will be received from a commercial party related directly or indirectly to the subject of this manuscript. This study is exempt from IRB approval, as it utilizes data from the deidentified ACS-NSQIP database.

\section{Conflict of Interest}

None declared

\section{References}

1 Singer A, Exuzides A, Spangler L, et al. Burden of illness for osteoporotic fractures compared with other serious diseases among postmenopausal women in the United States. Mayo Clin Proc 2015;90(1):53-62

2 Wasfie T, Jackson A, Brock C, Galovska S, McCullough JR, Burgess JA. Does a fracture liaison service program minimize recurrent fragility fractures in the elderly with osteoporotic vertebral compression fractures? Am J Surg 217(3):557-560

3 Lee BG, Choi JH, Kim DY, Choi WR, Lee SG, Kang CN. Risk factors for newly developed osteoporotic vertebral compression fractures following treatment for osteoporotic vertebral compression fractures. Spine J 2019;19(2):301-305

4 Fechtenbaum J, Etcheto A, Kolta S, Feydy A, Roux C, Briot K. Sagittal balance of the spine in patients with osteoporotic vertebral fractures. Osteoporos Int 2016;27(2):559-567

5 Zhang YL, Shi LT, Tang PF, Sun ZJ, Wang YH. Correlation analysis of osteoporotic vertebral compression fractures and spinal sagittal imbalance. Orthopade 2017;46(3):249-255

6 Li HM, Zhang RJ, Gao H, et al. New vertebral fractures after osteoporotic vertebral compression fracture between balloon kyphoplasty and nonsurgical treatment PRISMA. Medicine (Baltimore) 2018;97(40):e12666
7 Gupta A, Upadhyaya S, Cha T, Schwab J, Bono C, Hershman S. Serum albumin levels predict which patients are at increased risk for complications following surgical management of acute osteoporotic vertebral compression fractures. Spine J 2019;19(11):1796-1802

8 Yu WB, Jiang XB, Liang D, Xu WX, Ye LQ, Wang J. Risk factors and score for recollapse of the augmented vertebrae after percutaneous vertebroplasty in osteoporotic vertebral compression fractures. Osteoporos Int 2019;30(2):423-430

9 Shah LM, Jennings JW, Kirsch CFE, et al. Expert Panels on Neurological Imaging, Interventional Radiology, and Musculoskeletal Imaging. ACR Appropriateness Criteria Management of Vertebral Compression Fractures. J Am Coll Radiol 2018;15(11S) (11s):S347-S364

10 Yang W, Yang J, Liang M. Percutaneous vertebroplasty does not increase the incidence of new fractures in adjacent and nonadjacent vertebral bodies. Clin Spine Surg 2019;32(2):e99-e106

11 Schupfner R, Stoevelaar HJ, Blattert T, et al. Treatment of osteoporotic vertebral compression fractures: applicability of appropriateness criteria in clinical practice. Pain Physician 2016;19(1):e113-e120

12 Stone AV, Jinnah A, Wells BJ, et al. Nutritional markers may identify patients with greater risk of re-admission after geriatric hip fractures. Int Orthop 2018;42(2):231-238

13 Lau E, Ong K, Kurtz S, Schmier J, Edidin A. Mortality following the diagnosis of a vertebral compression fracture in the Medicare population. J Bone Joint Surg Am 2008;90(7):1479-1486

14 Chung AS, Hustedt JW, Walker R, Jones C, Lowe J, Russell GV. Increasing Severity of Malnutrition Is Associated With Poorer 30-Day Outcomes in Patients Undergoing Hip Fracture Surgery. J Orthop Trauma 2018;32(4):155-160

15 Goldstein CL, Chutkan NB, Choma TJ, Orr RD. Management of the elderly with vertebral compression fractures. Neurosurgery 2015;77(1(Suppl 4):S33-S45

16 Melton LJ III, Kan SH, Frye MA, Wahner HW, O’Fallon WM, Riggs BL. Epidemiology of vertebral fractures in women. Am J Epidemiol 1989;129(5):1000-1011

17 Silverman SL. The clinical consequences of vertebral compression fracture. Bone 1992;13(13(Suppl 2):S27-S31

18 Amin S, Achenbach SJ, Atkinson EJ. Khosla S, Melton LJ III. Trends in fracture incidence: a population-based study over 20 years. J Bone Miner Res 2014;29(3):581-589

19 Chen AT, Cohen DB, Skolasky RL. Impact of nonoperative treatment, vertebroplasty, and kyphoplasty on survival and morbidity after vertebral compression fracture in the medicare population. J Bone Joint Surg Am 2013;95(19):1729-1736

20 Goz V, Errico TJ, Weinreb JH, et al. Vertebroplasty and kyphoplasty: national outcomes and trends in utilization from 2005 through 2010. Spine J 2015;15(5):959-965

21 Lavelle EA, Cheney R, Lavelle WF. Mortality prediction in a vertebral compression fracture population: the ASA physical status score versus the Charlson comorbidity Index. Int J Spine Surg 2015;9:63

22 Toy JO, Basques BA, Grauer JN. Morbidity, mortality, and readmission after vertebral augmentation: analysis of 850 patients from the American College of Surgeons National Surgical Quality Improvement Program database. Spine 2014; 39(23):1943-1949

23 Mayhew D, Mendonca V, Murthy BVS. A review of ASA physical status - historical perspectives and modern developments. Anaesthesia 2019;74(3):373-379

24 Abouleish AE, Leib ML, Cohen NH. ASA provides examples to each ASA physical status class. ASA Newsl 2020;79(6):38-49

25 Hurwitz EE, Simon M, Vinta SR, et al. Adding examples to the ASA-physical status classification improves correct assignment to patients. Anesthesiology 2017;126(4):614-622 
26 Mac Grory B, Flood S, Schrag M, Paciaroni M, Yaghi S. Anticoagulation resumption after stroke from atrial fibrillation. Curr Atherosc Rep 2019;21(8):29

27 Guzik A, Bushnell C. Stroke epidemiology and risk factor management. Continuum (Minneap Minn) 2017;23(1, Cerebrovascular Disease) :15-39

28 Kai AM, Vadivelu N, Urman RD, Shukla S, Schonberger R, Banack T. Perioperative considerations in the management of anticoagulation therapy for patients undergoing surgery. Curr Pain Headache Rep 2019;23(2):13

29 Sugiyama M, Ueno Y, Kamo H, et al. Specific mechanisms of subarachnoid hemorrhage accompanied by ischemic stroke in essential thrombocythemia: two case reports and a literature review. J Neurol 2019;266(8):1869-1878

30 Batista TFP, Manuel PF, Correia AC. Essential thrombocythemia - A predisponent factor for stroke. Rev Assoc Med Brasil 2019;65(6):772-774

31 Oza R, Rundell K, Garcellano M. Recurrent ischemic stroke: strategies for prevention. Am Family Phys 2017;96(7):436-440

32 Liang SY. Sepsis and other infectious disease emergencies in the elderly. Emerg Med Clin North Am 2016;34(3):501-522

33 Rowe TA, McKoy JM. Sepsis in older adults. Infect Dis Clin North Am 2017;31(4):731-742

34 Moghadamyeghaneh Z, Hanna MH, Blondet JJ, et al. Impact of chronic steroid use on outcomes of colorectal surgery. Am J Surg 2015;210(6):1003-1009, discussion 1009

35 Singla A, Qureshi R, Chen DQ et al. Risk of surgical site infection and mortality following lumbar fusion surgery in patients with chronic steroid usage and chronic methicillin-resistant Staphylococcus aureus infection. Spine 2019;44(7):e408-e413

36 Ilyas H, Golubovsky JL, Chen J, Winkelman RD, Mroz TE, Steinmetz MP. Risk factors for 90-day reoperation and readmission after lumbar surgery for lumbar spinal stenosis. J Neurosurg Spine 2019;31(1):20-26

37 Elsamadicy AA, Adogwa O, Vuong VD, et al. Patient body mass index is an independent predictor of 30-day hospital readmission after elective spine surgery. World Neurosurg 2016;96(Dec):148-151

38 Behnke NK, Baker DK, Xu S, Niemeier TE, Watson SL, Ponce BA. Risk factors for same-admission mortality after pathologic fracture secondary to metastatic cancer. Support Care Cancer 2017;25(2):513-521

39 Charlson ME, Pompei P, Ales KL, MacKenzie CR. A new method of classifying prognostic comorbidity in longitudinal studies: development and validation. J Chronic Dis 1987; 40(5):373-383

40 Deyo RA, Cherkin DC, Ciol MA. Adapting a clinical comorbidity index for use with ICD-9-CM administrative databases. J Clin Epidemiol 1992;45(6):613-619

41 Ong Kl, Beall DP, Frohbergh M, Lau E, Hirsch JA. Were VCF patients at higher risk of mortality following the 2009 publication of the vertebroplasty "sham" trials? Osteopor Int 2018;29(2):375-383

42 Hirsch JA, Chandra RV, Carter NS, Beall D, Frohbergh M, Ong K. Number needed to treat with vertebral augmentation to save a life. AJNR Am J Neuroradiol 2020;41(1):178-182

43 Hinde K, Maingard J, Hirsch JA, Phan K, Asadi H, Chandra RV. Mortality outcomes of vertebral augmentation (vertebroplasty and/or balloon kyphoplasty) for osteoporotic vertebral compression fractures: a systematic review and meta-analysis. Radiology 2020;295(1):96-103

44 Gutman IM, Niemeier TE, Gilbert SR. National databases in pediatric orthopaedic surgery: a comparison of demographics, procedures, and outcomes. J Pediatr Orthop 2019;39(8):e636-e640

45 Eisenstein S, Stringfield S, Holubar SD. Using the National Surgical Quality Improvement Project (NSQIP) to perform clinical research in colon and rectal surgery. Clin Colon Rectal Surg 2019;32(1):41-53

46 Bernatz JT, Tueting JL, Anderson PA. Thirty-day readmission rates in orthopedics: a systematic review and meta-analysis. PLoS One 2015;10(4):e0123593 